\title{
Research Paper \\ Measuring operational efficiency in some sectors in the Iraqi budget for the period 2005-2019
}

Journal of

\section{TANMIYAT AL- RAFIDAIN}

\section{(TANRA)}

A scientific, quarterly, international, open access, and peer-reviewed journal

Vol. 40, No. 131

September 2021

(C) University of Mosul | College of Administration and Economics, Mosul, Iraq.

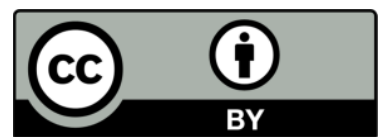

TANRA retains the copyright of published articles, which is released under a "Creative Commons Attribution License for CC-BY-4.0" enabling the unrestricted use, distribution, and reproduction of an article in any medium, provided that the original work is properly cited.

Citation: Ali, Oday Salim, Mohammad, Ahmed Jassem, (2021).“ Measuring operational efficiency in some sectors in the Iraqi budget for the period 20052019”. TANMIYAT ALRAFIDAIN, 40 (131), 315 -331, https://doi.org/

10.33899/tanra.2021.129846.10 83

P-ISSN: 1609-591X

e-ISSN: 2664-276X

tanmiyat.mosuljournals.com

\section{Oday Salim Ali ${ }^{1}$ Ahmed Jassem Mohammad ${ }^{2}$}

$1 \& 2$ Financial and Banking Sciences, College of Administration and EconomicsUniversity of Mosul.

Corresponding author: Oday Salim Ali, Financial and Banking Sciences, College of Administration and Economics-University of Mosul. odaysalimali@gmail.com

DOI: https://doi.org/ 10.33899/tanra.2021.129846.1083

Article History: Received:31/3/2021; Revised: 20/4/2121; Accepted: 28/4/2021; Published: 1/9/2021.

\section{Abstract}

There is no doubt that governments seek to achieve operational efficiency for any of the spending sectors, which is the efficiency that includes achieving maximum value for money and efficiency that aims to achieve the ultimate goals of government policy. In this research, the two researchers seek to measure the operational efficiency of some sectors of spending in Iraq, which are (investment spending (represented by spending on infrastructure), spending on education, and spending on health) during the period 2005-2019 using data envelope analysis that makes each year of the budget year a unit. make a decision. The research found that the efficiency of spending goes towards less spending in the budget, meaning that with the increase in spending, there is no improvement in performance indicators, meaning that the increase in spending on the studied sectors without leading to improvement in performance indicators indicates that there is great waste in spending on these Sectors. The study recommends the possibility of reducing spending instead of the volume of waste in spending, which gives the same performance results in the case of reduction, or the adoption of the budget of programs and performance to improve performance indicators of the ministries spending on them.

Keywords:

operational efficiency ' public spending ‘ spending efficiency. 


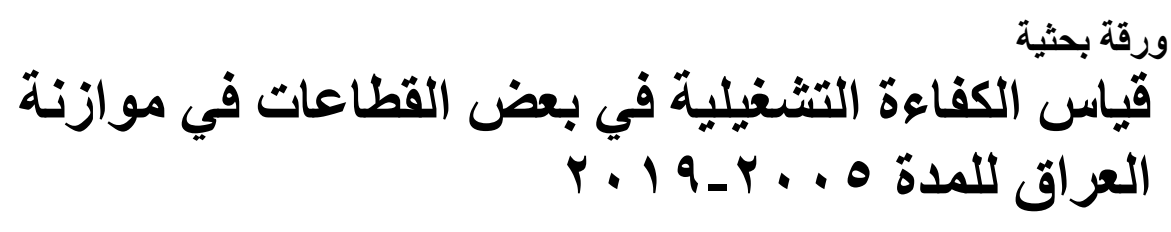

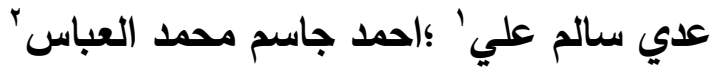

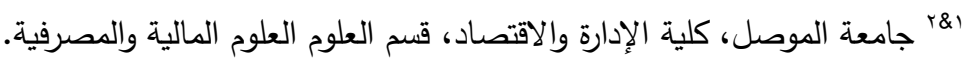

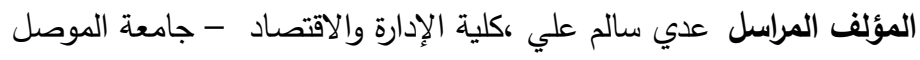
odaysalimali@gmail.com

DOI: https://doi.org/ 10.33899/tanra.2021.129846.1083

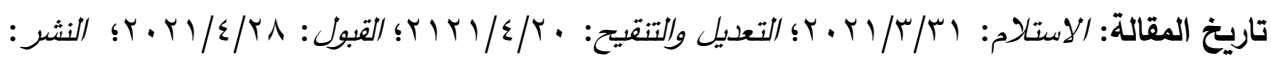

$$
\cdot r \cdot r / 9 / 1
$$

المستخلص

لا شك تسعى الحكومات إلى تحقيق الكفاءة التشغيلية لأي قطاع من قطاعات الإنفاق وهي الكفاءة التي

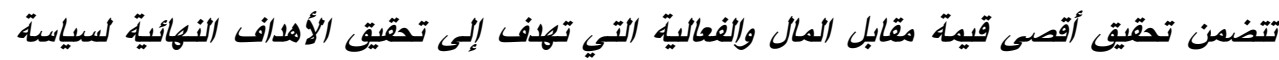
المكومة. وفي هذا البحث بسعى الباحثان لقياس الكفاءة التشغيلية لبعض قطاعات الإنفاق في العرلق

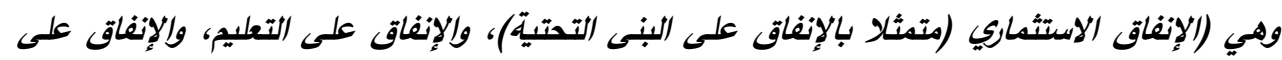

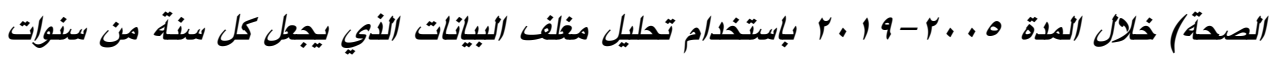

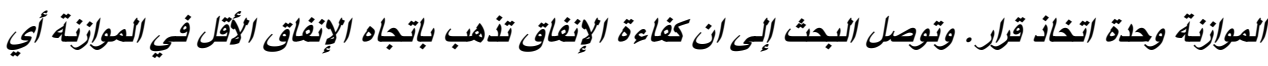

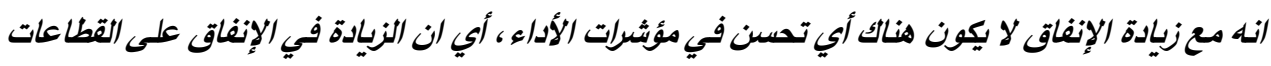

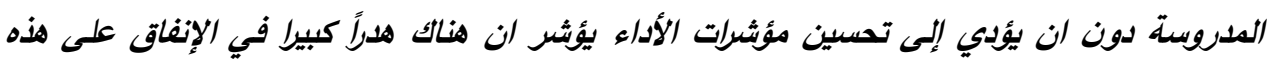

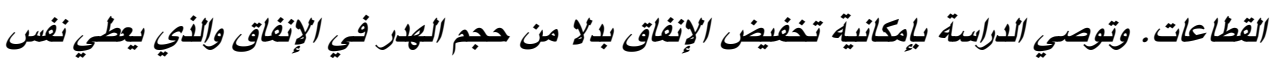

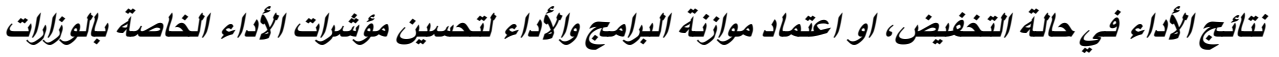
المنفق عليها.

الكلمات الرئيسة

الكفاءة التثغيلية ، الإنفاق العام ، كفاءة الإنفاق

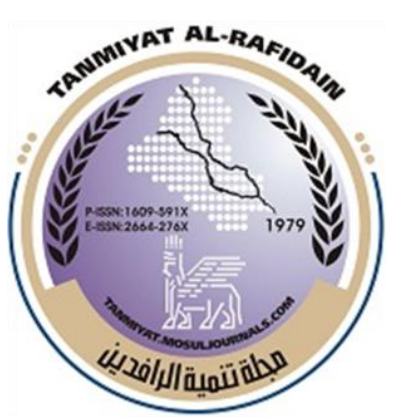

\section{هجلة}

\section{تنسية الرافدين}

(TANRA) دولية، مفتوحة الوصول، محكمة.

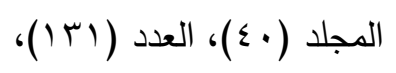

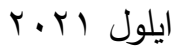

(ج) جامعة الموصل | كلية الإدارة والاقتصاد، الدوصل، العراق.

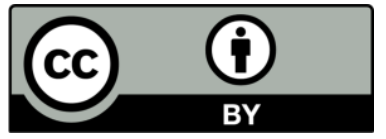

تحتفظ (TANRA) بحقوق الطبع والنشر للمقالات

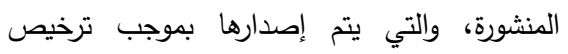

\lrcorner (Creative Commons Attribution) (CC-BY-4.0)

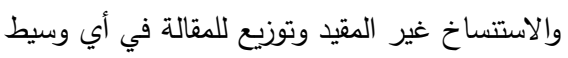
نقل، بشرط اقتباس العمل الأصلي بشكل صحيح.

الاقتباس: علي، عدي سالم، العباس، احمد

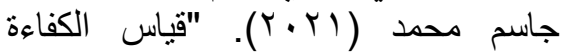
التشغيلية في بعض القطاعات في موازنة

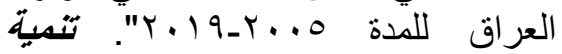

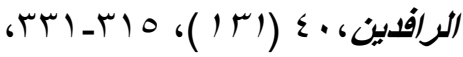
https://doi.org/ 10.33899/tanra.2021.129846.10 83

P-ISSN: 1609-591X

e-ISSN: 2664-276X

tanmiyat.mosuljournals.com 
تسعى الحكومات إلى تقديم الخدمات العامة، لأن منافع هذه الخدمات هي أحد أركان واجبات الدول،

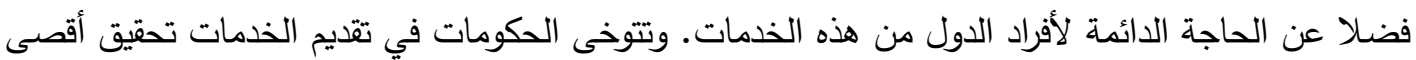
قدر من الكفاءة والفعالية نظرا لمحدودية الموارد فهي تبحث عن تحقيق أقصى قيمة مقابل وحدة نقدية ينفق.

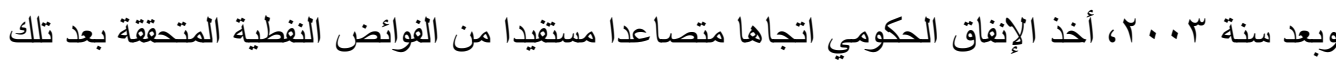

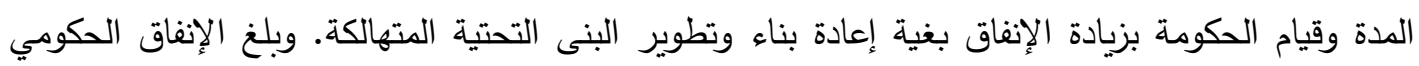

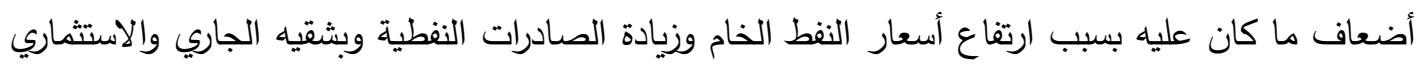
لأجل تحقيق الأثر المنشود من الإنفاق في جميع القطاعات المنفق عليها، ووصولها إلى الأفراد والأماكن التي

يسعى البحث إلى قياس كفاءة الإنفاق في ثلاثة قطاعات مهمة ذات تماس مع حياة الفرد العراقي، وهل تحققت كفاءة الإنفاق من خلال المنفعة المجمعية من هذه للأنثطة، والفعالية التي هي الأثر النهائي لهذا

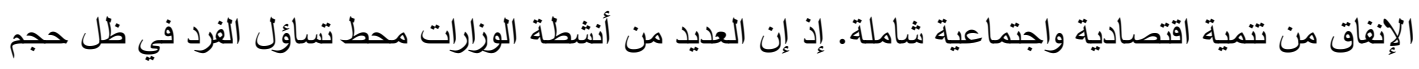

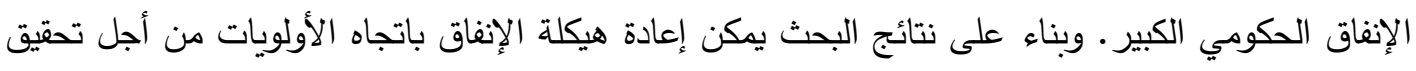
أقصى قدر من الكفاءة. مشكلة البحث

على الرغم من الفوائض النفطية المتحققة خلال المدة بعد ب . . ب، إلا أن العراق مر بأزمات مالية وسياسة خارجية وداخلية أثرت على العائدات النفطية. وبات من الضروري تحقيق كفاءة في كل أوجه الإنفاق، وهو مسؤولية كل حكومة وتتمثل مشكلة البحث في التساؤل الآتي: هل حقق الإنفاق على قطاعات (البنى التحتية، التعليم ،الصحة ) الكفاءة في الإنفاق ؟ فرضية البحث هن الإن

يفترض البحث أن هناك كفاءة في الإنفاق على قطاعات (البنى التحتية ، التعليم ،الصحة ) . هدف البحث

يهذف البحث إلى قياس كفاءة الإنفاق في ثلاثة قطاعات هي (الإنفاق الاستثماري (يمثل الإنفاق على

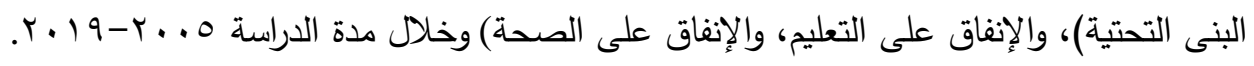
منهج البحث

اعتمد البحث أسلوب تحليل مغلف البيانات (Data Envelopment Analysis -DEA) الذي يقيس

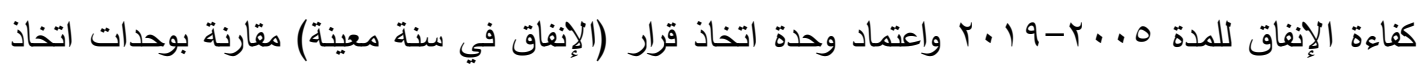
القرار الأخرى (الإنفاق بالسنوات الأخرى) • حيث يعطي هذا الأسلوب قيمة الواحد الصحيح لوحدة اتخاذ القرار الكفوءة، وأقل من واحد صحيح لوحدات اتخاذ القرار غير الكفوة. وتم اعتماد الإنفاق الاستثماري، لأنه يمثل لإنل 


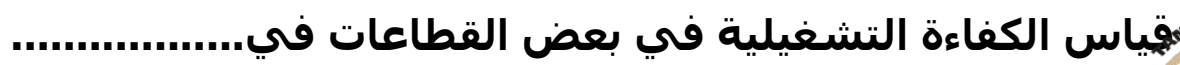 \\ علي والعباس}

الإنفاق على البنى التحتية، والإنفاق على التعليم والإنفاق على الصحة وربطها بمجموعة مؤشرات أداء لكل قطاع

1 ـ منهج الحكومة في تقديم الخدمات بكفاءة

1-1 ا لاكفاءة التشغيلية (تقديم الخدمات بكفاءة)

تتضمن الكفاءة التثغيلية تقديم الكيانات الادارية المختلفة مستوى محدد من الخدمات العامة باستخدام أقل

قدر ممكن من الموارد المالية، أو أن تتفق قدراً محدداً من الموارد لتقديم أفضل مستوى من الخدمات العادئ العامة

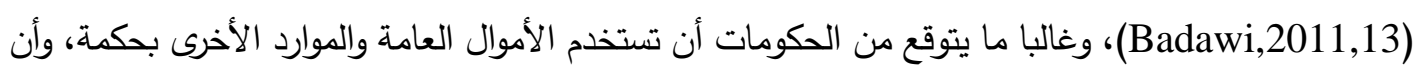
تعامل الجميع بإنصاف، ويجب أن تكون قادرة على تحقيق الاهداف العامة المطلوبة. ولهذا فان الحكومات دائما

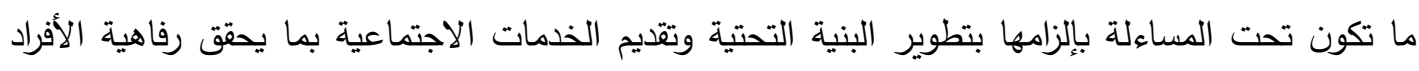

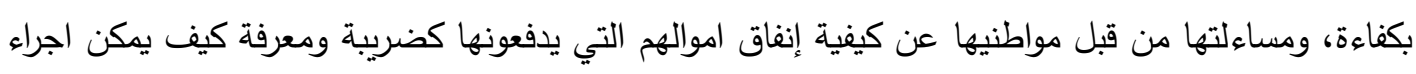
تطوير مستمر في جميع مجالات الإنفاق (Khalil \& Adelabu,2012,88 ). لا يملك غالبية السكان الوسائل الاقتصادية للوصول إلى السلع الأساسية من خلال الأسواق، مثل مياه

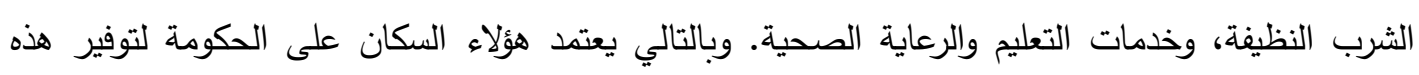

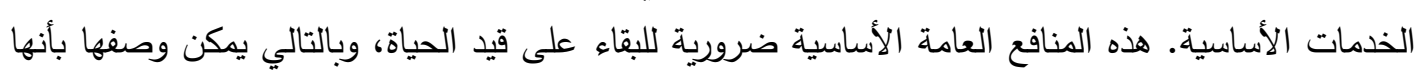

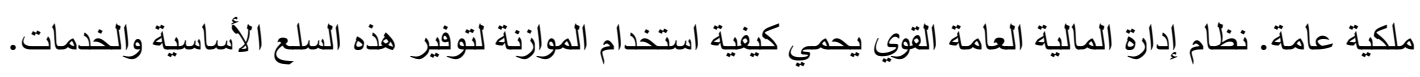
وبالتالي يسهم نظام إدارة المالية العامة الذي يعمل بثكل سليم في كفاءة المؤسسات، ويعكسها في الخدمات

.(Prakash \& Cabezon,2008,1)

وقد أدى الحد من الموارد والزيادة في طلب الأفراد على الخدمات وتنوعها إلى البحث عن استخدامات أكثر فعالية للأموال العامة (2018,4132, Sanchez \& Ballesteros). فضلا عن ذلك أدت الأزمات المالية

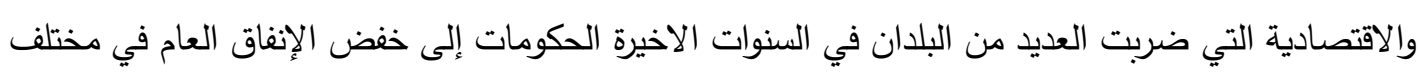

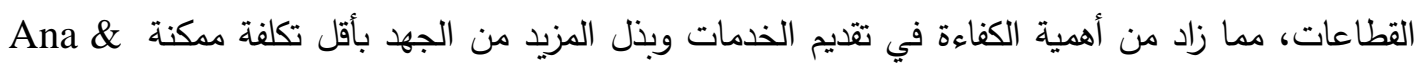
.(et.al ,2020,1)

يتم تحقيق هدف تقديم الخدمات العامة عندما تقدم الخدمة بكفاءة وفعالية اقتصادية على أساس الاحتياجات

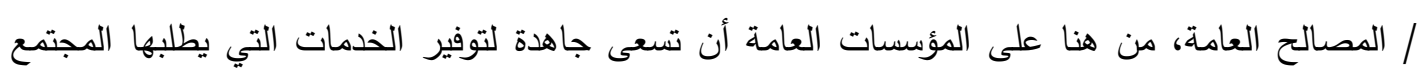
كمستهلك. وتقديم الخدمات بكفاءة يعني ضمناً تلبية أهداف تقديم الخدمات باستخدام الموارد الاقتصادية

.(Bekele, 2018, 18) r-r تحقيق القيمة مقابل المال في الإنفاق

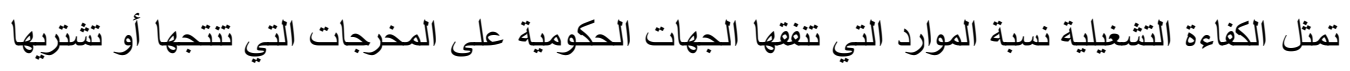

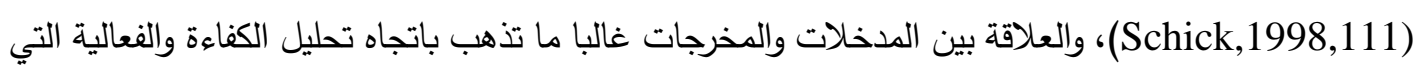
تحققها الحكومة، وهي تحقيق القيمة مقابل المال (Value For Money) أي القيمة التي تجنى مقابل المال 
الذي يتم إنفاقه، وهي تأمين أفضل مزيج من الكفاءة والفعالية بأقل نفقات على مدار مدة استخدام السلع او الخدمات المشتراة. أو هي الاستخدام الأمثل للموارد لتحقيق النتائج المرجوة (CDI,2015,2). وغالبا ما يتم التعبير عن القيمة مقابل المال من خلال ثلاثة معايير مختلفة هي (الاقتصاد والكفاءة

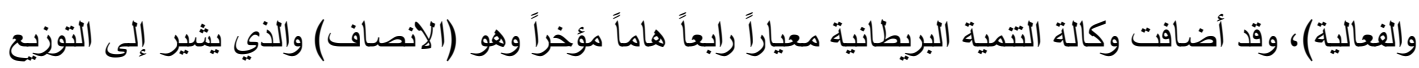
العادل للمنافع، وتعمل هذه المعايير بوصفها مبادئ يسترشد بها صناع القرار في إدارة المالية العامة، وتمثل

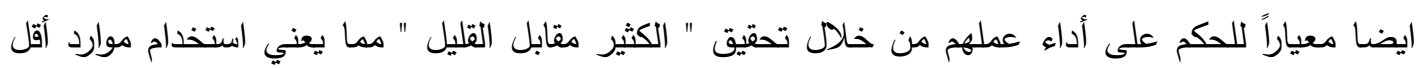
لتحقيق نتائج أفضل أو حتى النتائج نفسها ولضمان عملية تحسين مستمرة(Scholz,2020,1) . ويعكس الثكل (1) سلسلة القيمة مقابل المال للإنفاق على أي برنامج والتي تمثل مدخلات، ويهتم الاقتصاد بتكلفة وقيمة هذه المدخلات مع مراعاة النوعية، وتتعلق الكفاءة بالتكلفة الاجمالية للمدخلات التي يتم

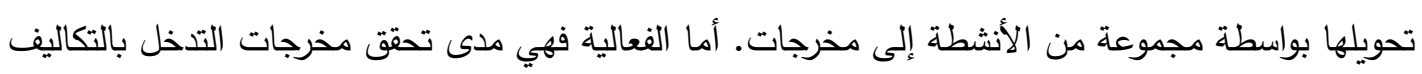
المنفقة النتيجة والأثر المرجو منها، والنتيجة والأثر التي تحصل عليها الحكومة تبرر التكاليف (CDI,2015,4).

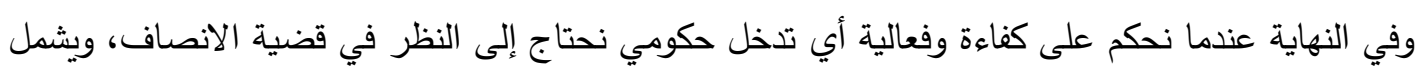

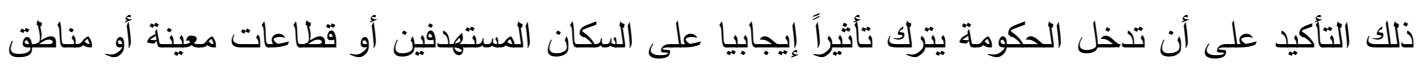

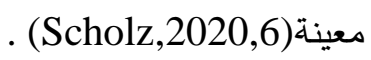

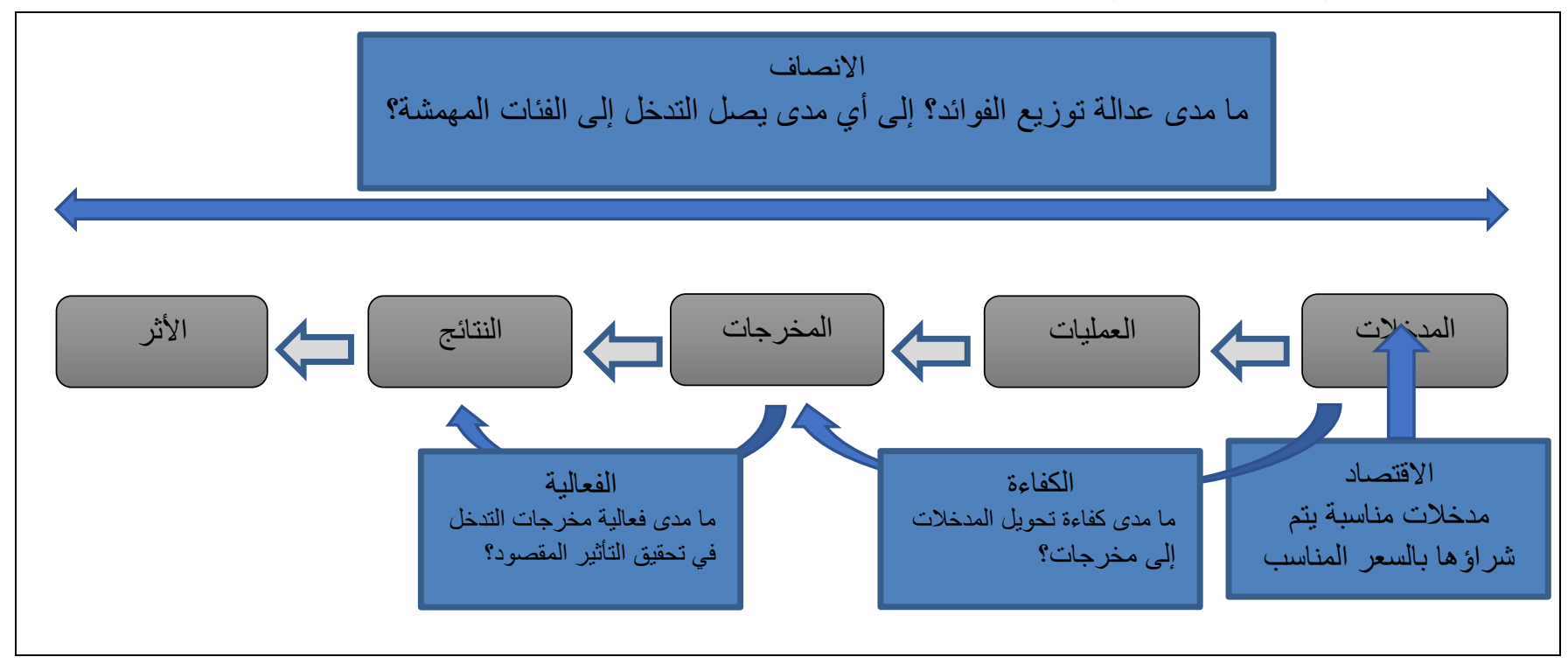

شكل (1) سلسلة تحقيق القيمة مقابل المال

Source: Scholz, Vera,2020, Value For Money , INTRAC for civil society,

p1..UK

وتدور القيمة مقابل المال حول تعظيم كل من الكفاءة والفعالية والاقتصاد بحيث يكون لدينا أقصى قدر من المبادئ الثلاثة لكل تدخل حكومي. ويوضح الثكل التالي كيف تحقق الحكومة أقصى كفاءة في الإنفاق، ونفترض إذا كان هناك ثلاثة بلدان "A" و "B" و "C" لهم المستوى نفسه من الإنفاق X1 الذي يمثل الدخخلات 
وتختلف الكفاءة فيما بينهم، ولكن البلد "A" يحقق أعلى ما يمكن من المخرجات Y1 وهي على حدود منحنى الكفاءة، بينما البلد "B" يحقق مستوى أقل من المخرجات بمقدار Y عن البلد "A" ، وبذلك يعد "A" أكثر كفاءة ، أما البلد "C" فيحقق القدر نفسه من المخرجات للبلد "B" بأقل ما يمكن من المدخلات، وهو بذلك يعد أكثر

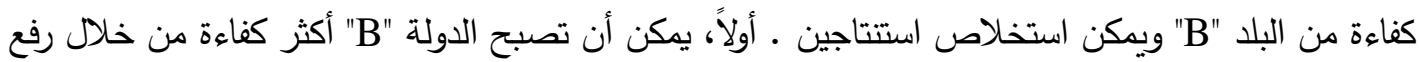

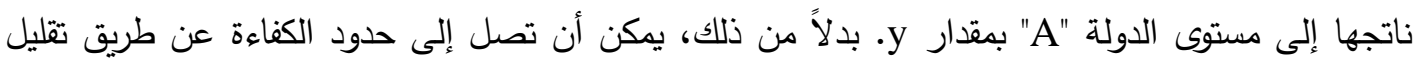
مدخلاتها بمقدار X إلى المستوى نفسه مثل البلد "C" (Mandl \& et.al,2008,8).

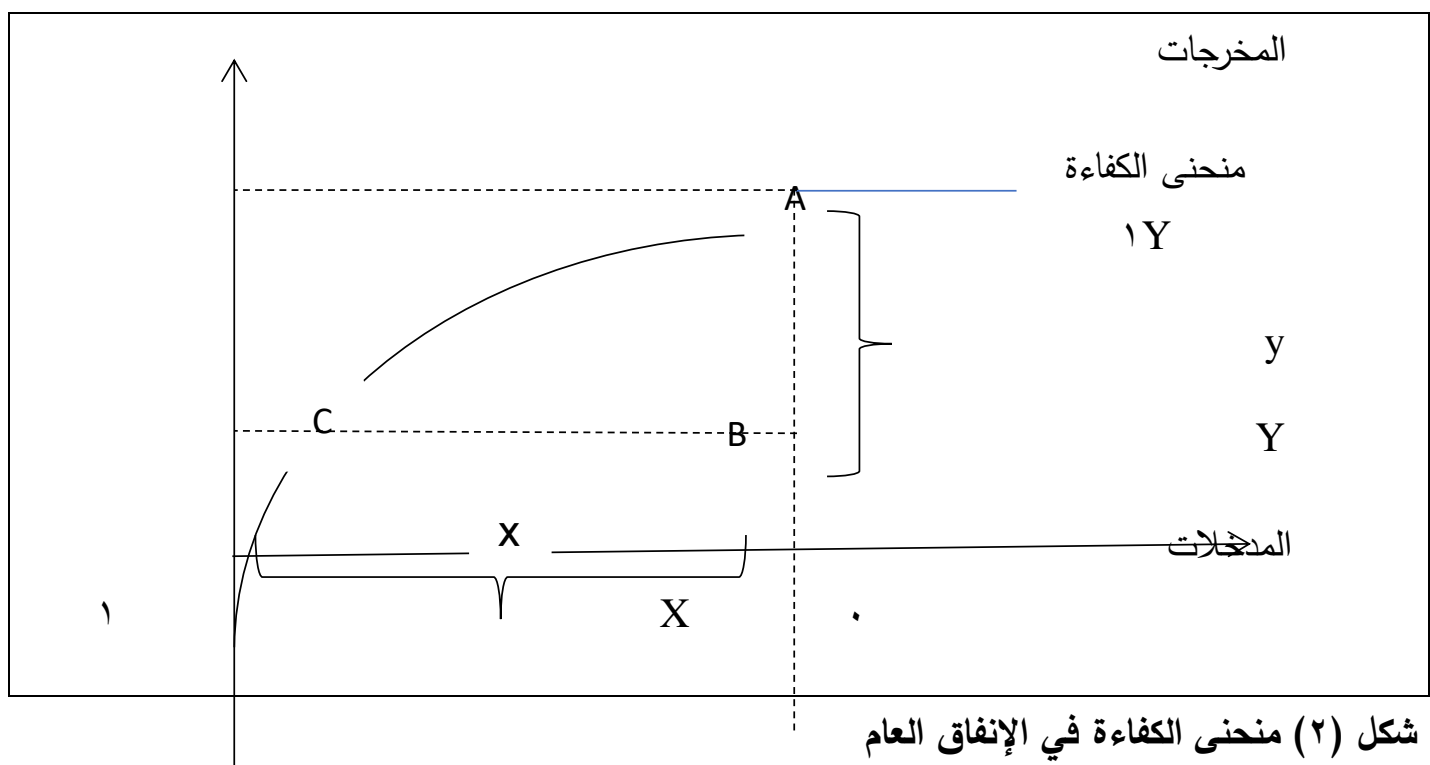

Source : Ulrike Mandl \& et.al ,2008, The Effectiveness and Efficiency of Public Spending, European Commission ,p8. مما سبق نستنتج أنه نظرا لمحدودية موارد الموازنة، ينبغي أن تحقق إدارة المالية العامة القيمة مقابل المال

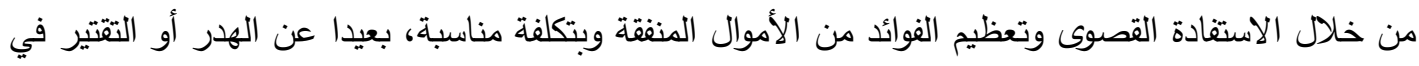

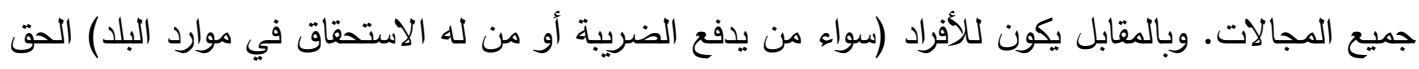
في الحصول على خدمات تعد ضرورية لحياتهم وبالنوعية الجيدة. ولا بد من أن يكون هناك توازن بين المبادئ الأربعة من أجل تحقيق القيمة مقابل المال فانتاج مخرجات

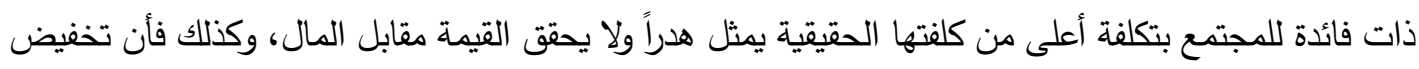
تكلفة برنامج أو مشروع معين من أجل توفير الأموال يؤثر على النتائج النهائية لا يمثل قيمة مقابل لئل المال.

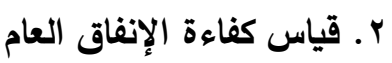
r-1 ـ ـ بمهيد لمنهج قياس كفاءة الإنفاق العام اكتسب قياس كفاءة الإنفاق قيمة كبيرة بالنسبة للحكومات، لأن الإنفاق العام يشكل نسبة كبيرة من إجمالي

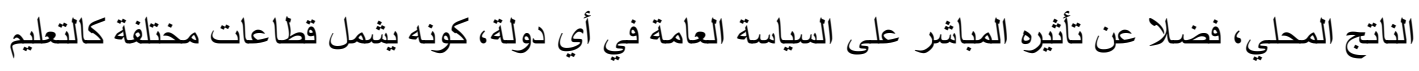


والرعاية الصحية والنقل والأمن العام. وبالنظر إلى مقدار الأموال التي تتفتها الحكومات كل عام على هذه الخدمات وغيرها، فانه ليس من الغرابة حصول أداء هذا الإنفاق على الكثير من الاهتمام على مر السنين السابقة، خاصة من قبل الجهات التي تقدم هذه الخدمات، وكذلك أفراد الدولة والمسؤولين المنتخبين أيضاً .(Khan \& Murova ,2015,170)

وعليه فإنه يتم تفسير المسافة النسبية إلى الحدود المقدرة لمغلف البيانات على أنها مقياس لمكاسب الكفاءة

التي يتم تحقيقها. عند رسم المدخلات على المحور السيني والمخرجات على المحور الصادي، فالمسافة العمودية من حد الكفاءة توضح إلى أي مدى يمكن توسيع الإنتاج مع الحفاظ على ثبات المدخلات وتمثل هذه المسافة (عدم كفاءة المخرجات). وبالمثل، فان المسافة الافقية من الحدود تقيس مدى إمكانية تقليل المدخلات دون التأثير على المخرجات أي (عدم كفاءة المدخلات) (Dutu \& Sicari 2020,255) كما يوضحها الثكل

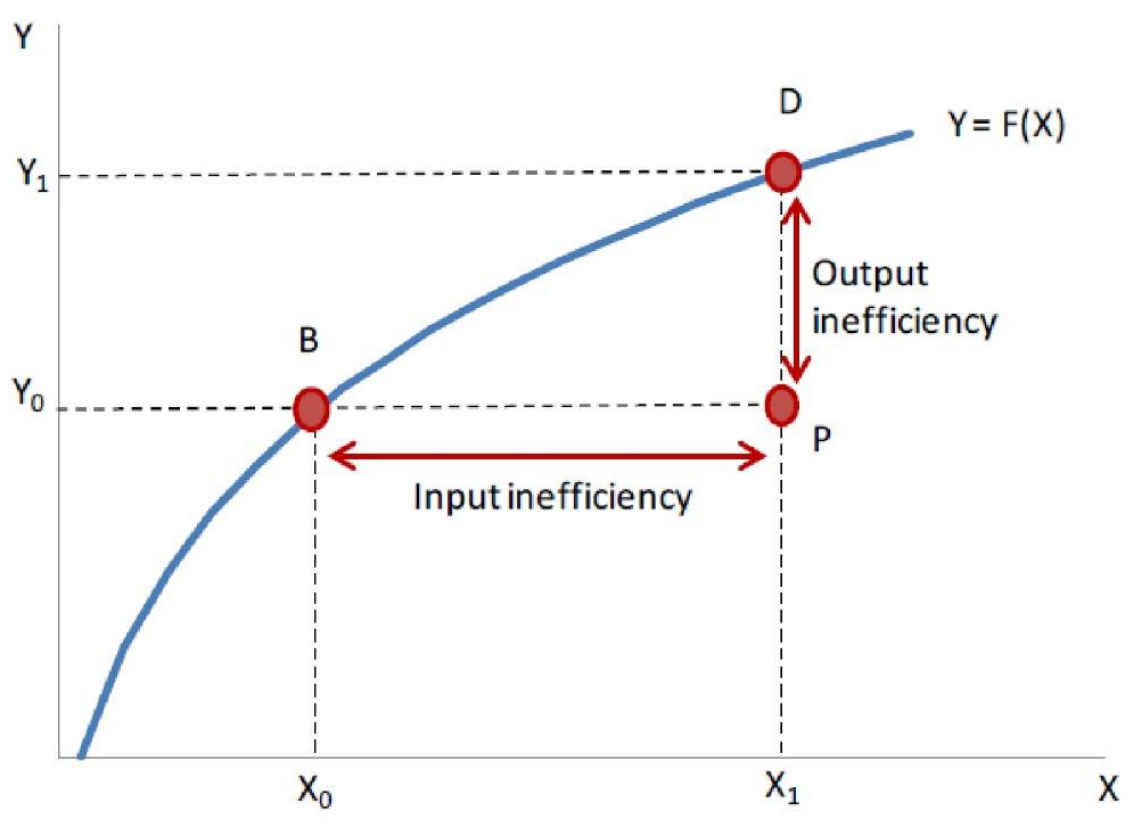

$$
\text { شكل (r ) حدود الكفاءة }
$$

Source: Dutu \& Sicari, Richard, Patrizio, 2020, Public Spending Efficiency in the OECD: Benchmarking Health Care, Education, and General Administration, Review of Economic Perspectives, Vol. 20, No 3.p255.

(Data Envelopment Analysis -DEA) r- r تحليل مغلف البيانات

يعد تحليل مغلف البيانات من الطرائق الثائعة لتقييم الكفاءة لكيانات الأعمال خاصة كانت أو عامة. ومغلف البيانات تقنية برمجة رياضية تتيح تحديد كفاءة الكيان بناءً على مدخلاتها ومخرجاتها، وتقارنها بالوحدات 


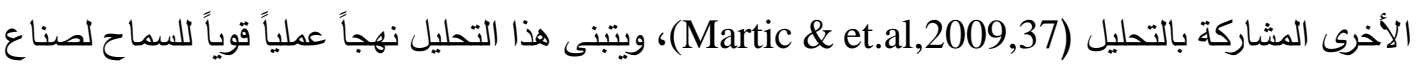
القرار بإجراء تحليل الكفاءة الخاص بهم وتفسير النتائج بسهولة (Huguenin,2012,5). تحليل مغلف البيانات (DEA) هو نهج جديد نسبيًا "موجه نحو البيانات" لتقييم أداء مجموعة من الكيانات

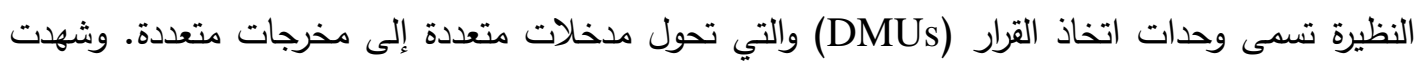
السنوات الأخيرة مجموعة كبيرة ومتنوعة من تطبيقات DEA لاستخدامها في تقييم أداء العديد من الأنواع

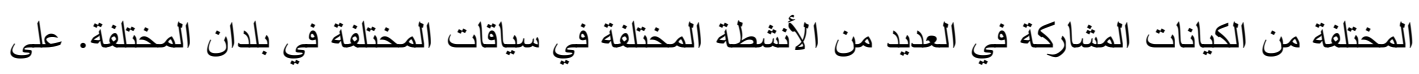
سبيل المثال، المستشفيات والجامعات والمدن والمحاكم وشركات الأعمال وغيرها، بما في ذلك أداء البلدان والمناطق وما إلى ذلك (Cooper \& et.al,2011,2) . إن DEA هو نهج غير معلمي يعتمد على البيانات، ويستخدم تقنيات البرمجة الخطية لحساب درجات الكفاءة لكل وحدة DMU في مجموعة بيانات يستوعب DEA مدخلات ومخرجات متعددة بسهولة دون الحاجة إلى قاسم مشترك للقياس. هذا يجعلها مناسبة بشكل خاص لتحليل كفاءة الكيانات الصحية والتعليمية، لأنها

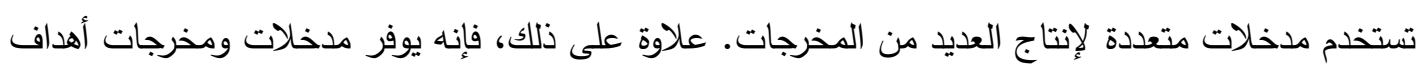
محددة من شأنها أن تجعل الكيانات الصحية والتعليمية غير الفعالة فعالة نسبيًا. كما أنه يحدد الأقران الأكفاء

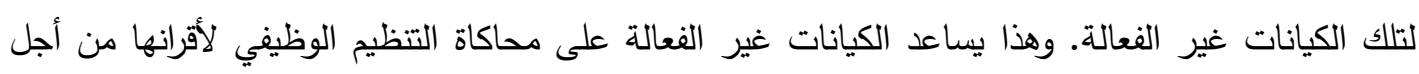
تحسين كفاءتها (Mujasi \& et.al,2016,4). يتم حساب درجة كفاءة كل كيان بالنسبة إلى حد الكفاءة. وبالتالي فإن الكيانات الواقعة على حدود الكفاءة

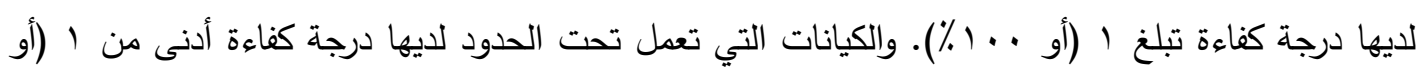

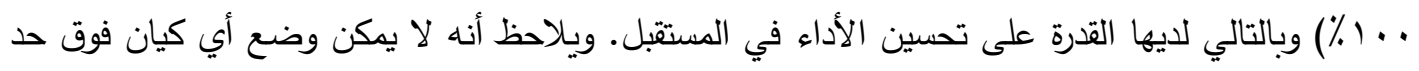

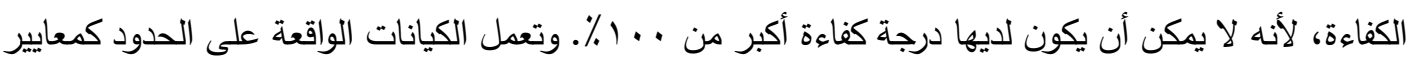

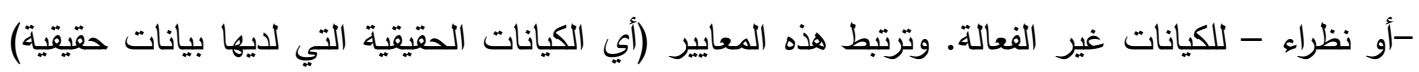
بأفضل الممارسات. ولذلك فإن تحليل مغلف البيانات يعد تقنية قياس مرجعية قوية. (Huguenin,2012,6)

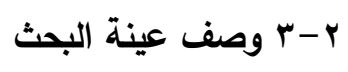
لغرض قياس الكفاءة التثغيلية لبعض الخدمات في العراق تم اعتماد ثلاثة مجالات للإنفاق يقابلها أربعة

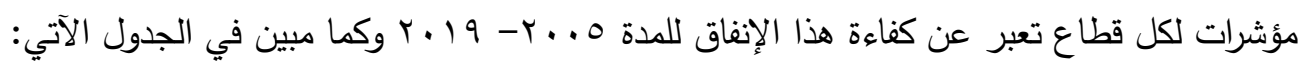


جدول (1) بعض قطاعات الإنفاق ومؤشرات أدائها

\begin{tabular}{|c|c|c|}
\hline 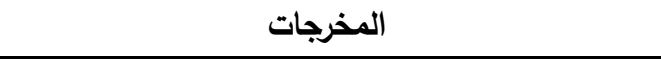 & 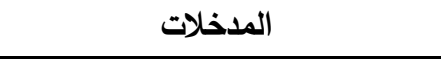 & القطاع \\
\hline الأراضي الزراعية (\% من مساحة الأراضي) & \multirow{4}{*}{ 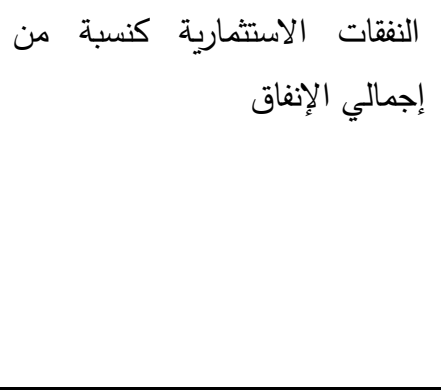 } & \multirow[t]{4}{*}{ البنى التحتية } \\
\hline نصيب الفرد من الكهرباء المتاحة (ميكا/ واط/ ساعة) & & \\
\hline 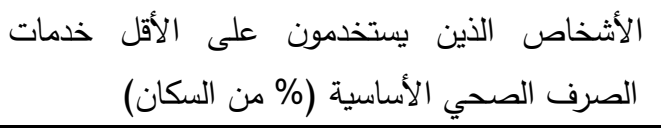 & & \\
\hline 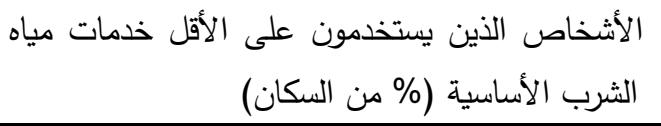 & & \\
\hline المعدل الصافي للالتحاق بالتعليم الابتدائي & \multirow{4}{*}{ إلجمالي الإنفاق على التعليم كنسبة من } & \multirow[t]{4}{*}{ 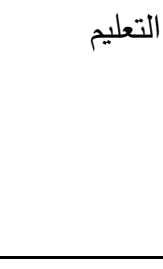 } \\
\hline عدد المقالات المنشورة & & \\
\hline معدل الالمام بالقراءة والكتابة لدى السكان البالغين & & \\
\hline المعدل الصافي للالتحاق بالتعليم الثانوي & & \\
\hline معتوفٍ لكل وفيات الأطفال دون الخامسة من العمر / طفل & \multirow[t]{4}{*}{ إلجمالي الإنفاق على الصحة كنسبة من } & \multirow[t]{4}{*}{ 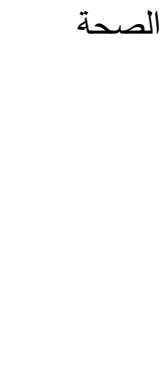 } \\
\hline العمر المتوقع عند الميلاد، إجمالي (بالسنوات) & & \\
\hline الرعاية الصحية الصئوية للسكان الذين يحصلون على خدمات & & \\
\hline أسِِرَّة المستثفيات (لكل ... . . شخص) & & \\
\hline
\end{tabular}

القطاعات والمؤشرات المختارة بناءً على الدراسات الآتية (Herrera \& Ouedraogo,2018,81-82) (Dutu \& Sicari) (Brini \& Jemmali,2016,27) (Afonso \& Kazemi ,2016,7) 2020,277-280 1- النفقات الاستثمارية كنسبة من إجمالي الإنفاق: تم استخدام هذا المؤشر كمعبر عن خدمات البنى التحتية

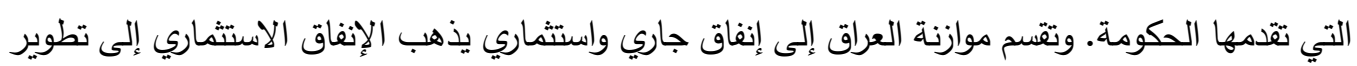

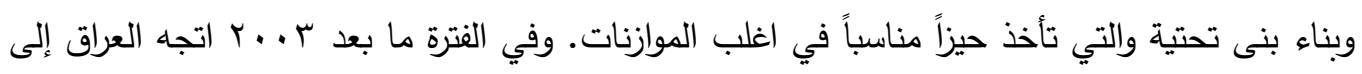

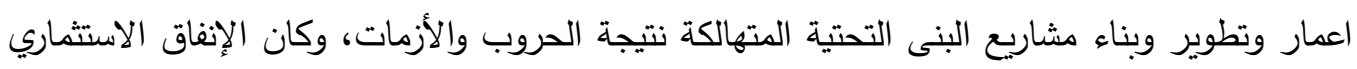
الرافد الرئيس لهذه المشاريع مستفيدا من الفوائض النفطية المتحققة للموازنة.

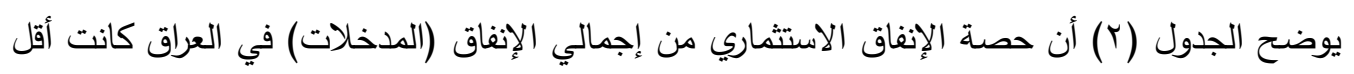

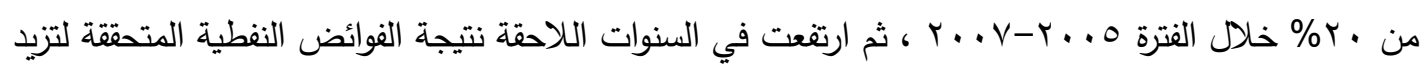

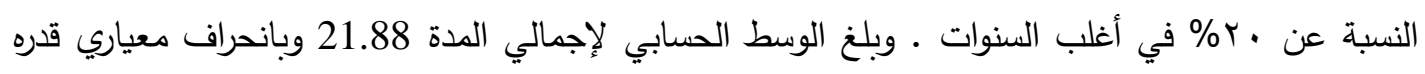

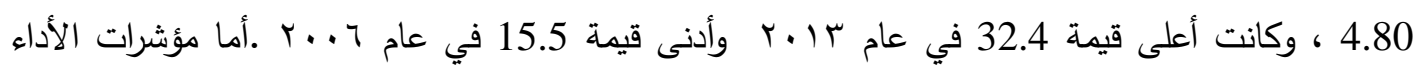

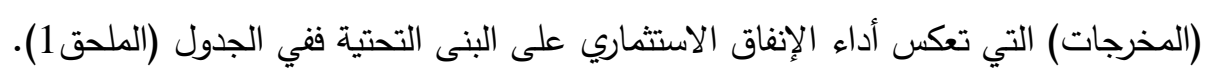


على والعباس

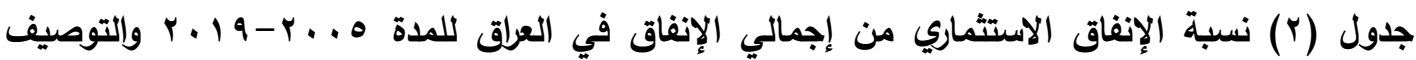

\begin{tabular}{|c|c|c|c|c|c|}
\hline & & & & \multicolumn{2}{|c|}{ الاحصائي } \\
\hline & & $\begin{array}{c}\text { نسبة إلى إجمالي الإنفاق الاستثمارية } \\
\text { (النفات } \\
\text { (\%) }\end{array}$ & إجمالي الإنفاق & 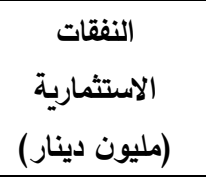 & السنة \\
\hline & & 17.3 & $26,375,175$ & $4,572,018$ & 2005 \\
\hline & & 15.5 & $38,806,679$ & $6,027,680$ & 2006 \\
\hline & & 16.8 & $39,308,349$ & $6,588,512$ & 2007 \\
\hline & & 20.0 & $59,403,375$ & $11,880,675$ & 2008 \\
\hline الاستثماري & توصيف نسبا & 20.0 & $52,567,025$ & $10,513,405$ & 2009 \\
\hline 21.88 & الوسط الحسابي & 23.0 & $64,351,984$ & $14,800,956$ & 2010 \\
\hline 4.80 & الانحراف المعياري & 19.6 & $69,639,523$ & $13,622,509$ & 2011 \\
\hline 21.81 & الوسيط & 23.0 & $90,374,783$ & $20,755,500$ & 2012 \\
\hline 32.4 & اعلى قيمة & 32.4 & $106,873,027$ & $34,647,008$ & 2013 \\
\hline 15.5 & أدني قيمة & 29.8 & $83,556,226$ & $24,930,767$ & 2014 \\
\hline 15 & المشاهدات & 26.4 & $70,397,515$ & $18,564,676$ & 2015 \\
\hline & & 23.7 & $67,067,437$ & $15,894,009$ & 2016 \\
\hline & & 21.8 & $75,490,115$ & $16,464,461$ & 2017 \\
\hline & & 17.1 & $80,873,189$ & $13,820,333$ & 2018 \\
\hline & & 21.9 & $111,723,522$ & $24,422,590$ & 2019 \\
\hline
\end{tabular}

المصدر: احتساب الباحث بالاعتماد على:

The Central Bank of Iraq, Annual Statistical Bulletin, various issues Ministry of Finance, Budget Department.

ץ- الإنفاق على التعليم كنسبة من إجمالي الإنفاق: تكرس الحكومات الوقت والأموال من اجل الاستثمار في

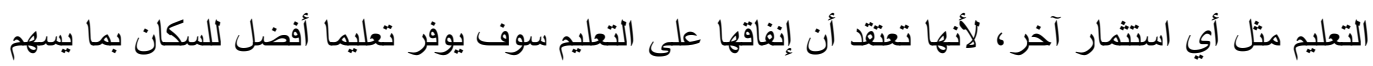

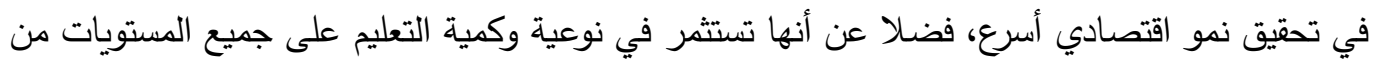
أجل توفير المساواة والحد من التفاوت في الدخل (Ali,2019,58). يتمثل الإنفاق على التعليم في العراق بالإنفاق على وزارتي التعليم العالي والبحث العلمي بالإضافة إلى الإنفاق على وزارة التربية.

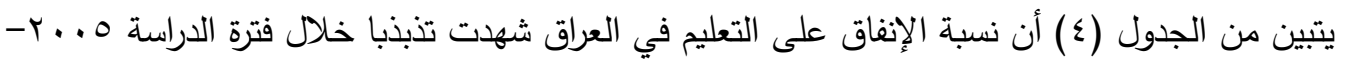

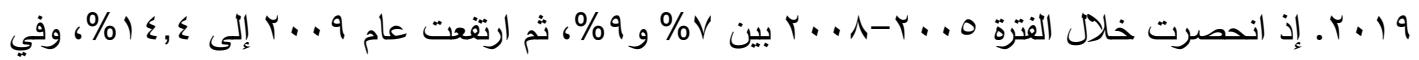

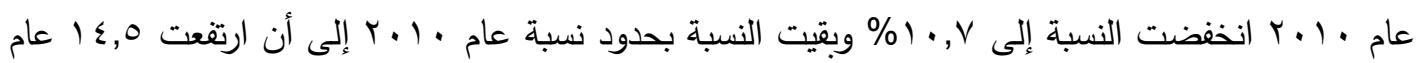

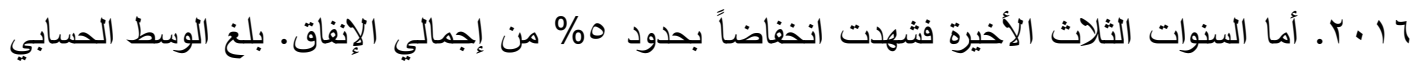




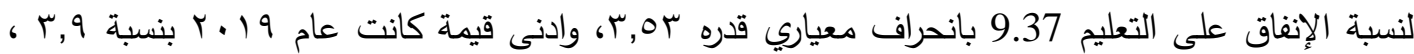

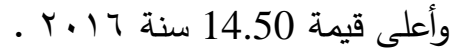

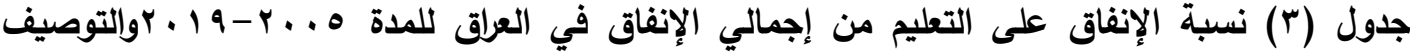

\begin{tabular}{|c|c|c|c|c|c|}
\hline & & & & \multicolumn{2}{|c|}{ الاحصائي } \\
\hline & & الإنفاق على التعليم & (مليون دينار) & التعليم(مليون & السنة/البند \\
\hline & & 7.0 & $26,375,175$ & $1,848,423$ & 2005 \\
\hline & & 5.6 & $38,806,679$ & $2,168,903$ & 2006 \\
\hline & & 7.3 & $39,308,349$ & $2,856,035$ & 2007 \\
\hline & & 9.0 & $59,403,375$ & $5,351,738$ & 2008 \\
\hline & التوصيف الا & 14.4 & $52,567,025$ & $7,582,669$ & 2009 \\
\hline 9.37 & الوسط الحسابي & 10.7 & $64,351,984$ & $6,888,873$ & 2010 \\
\hline$r 3.5$ & الانحراف المعياري & 11.6 & $69,639,523$ & $8,077,785$ & 2011 \\
\hline 10.10 & الوسيط & 10.7 & $90,374,783$ & $9,673,083$ & 2012 \\
\hline 14.50 & أعلى قيمة & 10.1 & $106,873,027$ & $10,787,831$ & 2013 \\
\hline 3.90 & أدني قيمة & 12.1 & $83,556,226$ & $10,087,317$ & 2014 \\
\hline 15 & المشاهدات & 13.4 & $70,397,515$ & $9,454,159$ & 2015 \\
\hline & & 14.5 & $67,067,437$ & $9,732,368$ & 2016 \\
\hline & & 5.3 & $75,490,115$ & $4,027,563$ & 2017 \\
\hline & & 5.0 & $80,873,189$ & $4,065,914$ & 2018 \\
\hline & & 3.9 & $111,723,522$ & $4,349,706$ & 2019 \\
\hline
\end{tabular}

المصدر: احتساب الباحث بالاعتماد على:

The Central Bank of Iraq, Annual Statistical Bulletin, various issues Ministry of Finance, Budget Department.

أما مؤشرات الأداء (المخرجات ) التي تعكس أداء الإنفاق على التعليم فتظهر في الجدول (ץ الملحق).

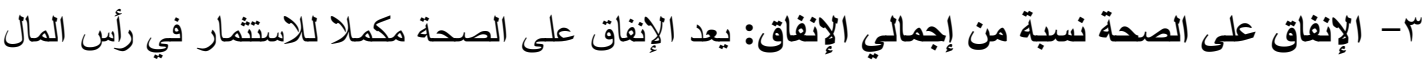
البشري إلى جانب الإنفاق على التعليم. والإنفاق على الخدمات الصحية حق أساسي من حن حقوق الإنى الإنسان

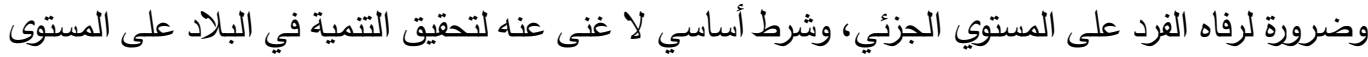

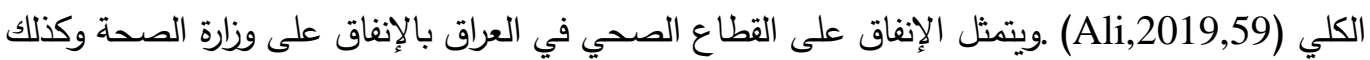
على وزارة البيئة التي ادمجت مؤخراً مع وزارة الصحة.

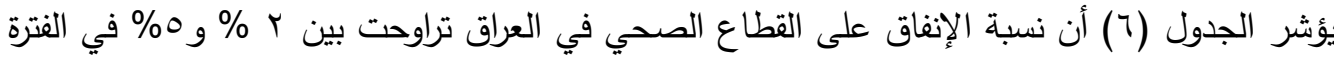

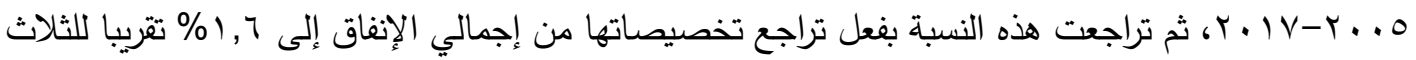

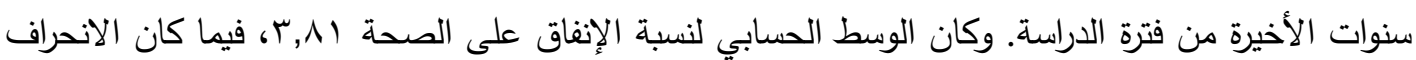




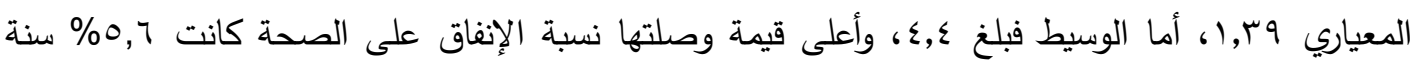

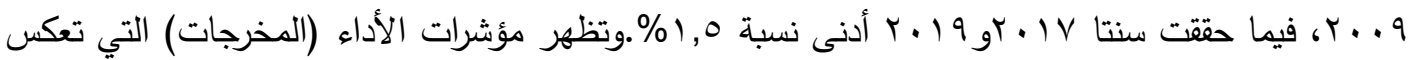
أداء الإنفاق على التعليم في الجدول (r الملحق).

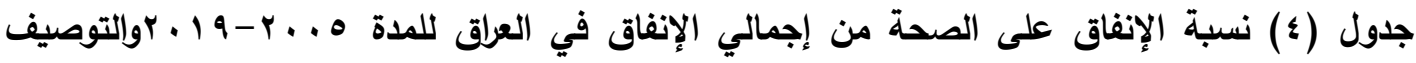

\begin{tabular}{|c|c|c|c|c|c|}
\hline & & & & \multicolumn{2}{|c|}{ الاحصائي } \\
\hline & & الإنفاق على الصحة إلى إجمالي & إجمالي النفقات & $\begin{array}{c}\text { (الإنفاق على } \\
\text { (مليون دينار) }\end{array}$ & السنة/البند \\
\hline & & 2.8 & $26,375,175$ & 728,116 & 2005 \\
\hline & & 2.9 & $38,806,679$ & $1,128,921$ & 2006 \\
\hline & & 4.3 & $39,308,349$ & $1,676,684$ & 2007 \\
\hline & & 3.8 & $59,403,375$ & $2,251,281$ & 2008 \\
\hline \multicolumn{2}{|c|}{ التوصيف الاحصائي } & 5.6 & $52,567,025$ & $2,938,220$ & 2009 \\
\hline 3.81 & الوسط الحسابي & 4.9 & $64,351,984$ & $3,137,096$ & 2010 \\
\hline 1.395 & الانحراف المعياري & 4.9 & $69,639,523$ & $3,406,172$ & 2011 \\
\hline 4.40 & الوسيط & 4.4 & $90,374,783$ & $3,956,990$ & 2012 \\
\hline 5.60 & أعلى قيمة & 4.5 & $106,873,027$ & $4,767,103$ & 2013 \\
\hline 1.50 & أدنى قيمة & 5.0 & $83,556,226$ & $4,147,176$ & 2014 \\
\hline 15 & المشاهدات & 4.7 & $70,397,515$ & $3,289,603$ & 2015 \\
\hline & & 4.8 & $67,067,437$ & $3,203,298$ & 2016 \\
\hline & & 1.5 & $75,490,115$ & $1,143,731$ & 2017 \\
\hline & & 1.6 & $80,873,189$ & $1,317,937$ & 2018 \\
\hline & & 1.5 & $111,723,522$ & $1,665,899$ & 2019 \\
\hline
\end{tabular}

المصدر: احتساب الباحث بالاعتماد على:

The Central Bank of Iraq, Annual Statistical Bulletin, various issues

Ministry of Finance, Budget Department.

r. بناء الأنموذج وتفسير النتائج

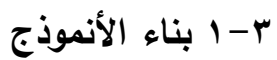

لغرض قياس الكفاءة التشغيلية لنسب الإنفاق الاستثماري والإنفاق على التعليم والإنفاق على الصحة على مخرجات كل قطاع من القطاعات، تم تحليل كفاءة كل سنة من السنوات 2005 ,.... 2019 لكل من تخصيصات القطاعات الثلاثة بأسلوب مغلف البيانات Data Envelopment Analysis DEA. إذ تم اعتبار كل سنة من هذه السنوات وحدة اتخاذ قرار Decision Making Unit DMUa، حيث إن ( ,..., 2019 
علي والعباس

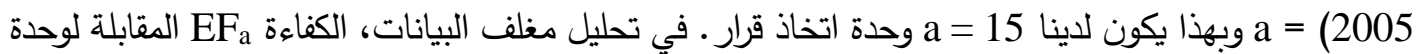

اتخاذ القرار DMU وتعرف على أنها نسبة مجموع المخرجات الموزونة إلى نسبة مجموع المدخلات الموزونة

$$
E F a=\frac{\sum_{i=1}^{N} \alpha_{i, a} Y_{i, a}}{\sum_{j=1}^{M} \beta_{j, a} X_{j, a}}
$$

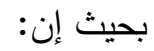

عندما:

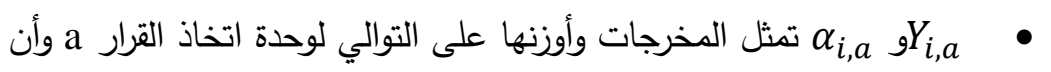

$$
\text { • }
$$

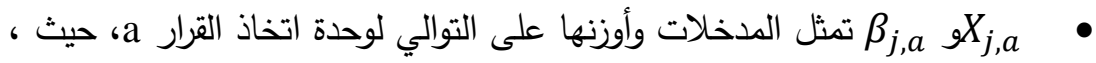

$$
\text { • }
$$

والمقياس المذكور آنفاً يمثل مقياس كفاءة وحدة اتخاذ القرار للسنة a في حال كان هناك وحدة اتخاذ قرار

واحدة. أما إذا كان هناك مجموعة من وحدات اتخاذ القرار، والهدف هو استخدام وحدات اتخاذ القرار العالية الكفاءة على انها الدليل في تعديل تخصيصات الوحدات غير الكفؤة، فأنه يجب أن يخضع هذا الفوان المقياس للقيود:

$$
a_{i, a}, \beta_{j, a}>0
$$

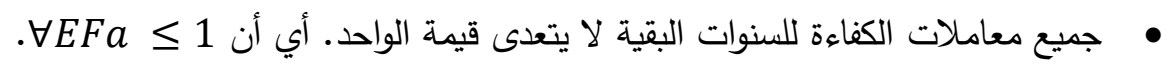

• والقيد الثالث هو أن القيم

$$
\text { غير منتهِ من الحلول. }
$$

• • عليه فإن أنموذج البرمجة الخطية سوف يكون بالصيغة الآتية:

$$
\max E F_{a}=\frac{\sum_{i=1}^{N} \alpha_{i, a} Y_{i, a}}{\sum_{j=1}^{M} \beta_{j, a} X_{j, a}}
$$

$$
E F_{K} \frac{\sum_{i=1}^{N} \alpha_{i, a} Y_{i, a}}{\sum_{j=1}^{M} \beta_{j, a} X_{j, a}} \leq 1
$$

$$
\sum_{j=1}^{M} \beta_{i, k} X_{i, k}=1
$$

يمكن حل البرمجة الخطية الكسرية المذكورة آنفاً بعد تحويلها إلى برمجة خطية تقليدية وبحسب الأنموذج

(Zhang \& et.al,2014,2-3) (Bowlin,1998,4-8)

$$
\max E F_{a}=\sum_{i=1}^{N} \alpha_{i, a} Y_{i, a}
$$




$$
\begin{aligned}
& \sum_{j=1}^{M} \beta_{i, k} X_{i, k}=1 \\
& \sum_{j=1}^{M} \beta_{i, k} X_{i, k}-\sum_{i=1}^{N} \alpha_{i, a} Y_{i, k} \leq 0 \\
& a_{i, a}, \beta_{j, a}>0 \quad i=1, \ldots N, j=1, \ldots, M \\
& \text { حيث إن k تمثل جميع وحدات اتخاذ القرار ما عدا } a .
\end{aligned}
$$

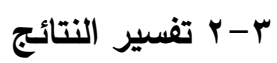

1 - كفاءة النفقات الاستثمارية نسبة من إجمالي الإنفاق: من خلال تطبيق أنموذج مغلف البيانات على نلى

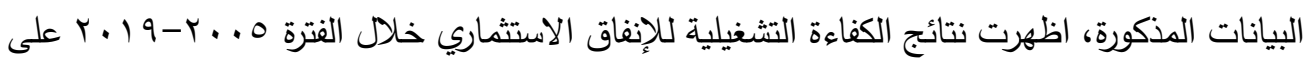
اعتبار أن كل سنة هي وحدة اتخاذ قرار الموضحة في الجدول (ع الملحق) أن هناك كفاءة للإنفاق

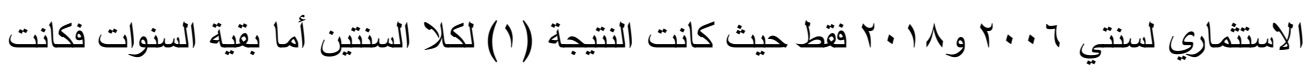

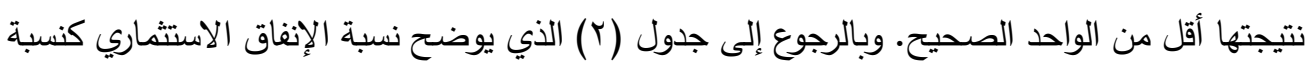

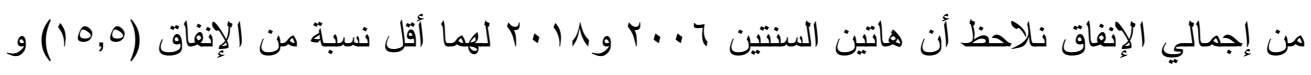
(lV, (1) فإن الزيادة في النسب في بقية السنوات أي زيادة في التخصيصات الاستثمارية لم تؤدِ إلى تحسين مؤشرات الأداء للمتغيرات المختارة وتعطي النتيجة نفسها، أي إن هذئ فأن الأموال تمثل هدراً في التخصيصات لأنها تعطي نتائج الاداء نفسه بنسب أقل (بتخصيصات أعضات أقل).

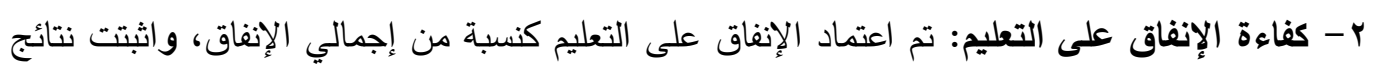

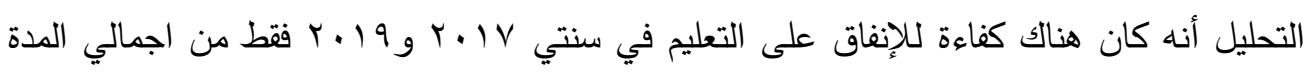

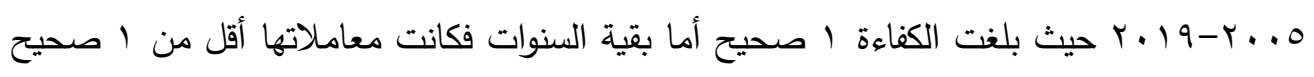

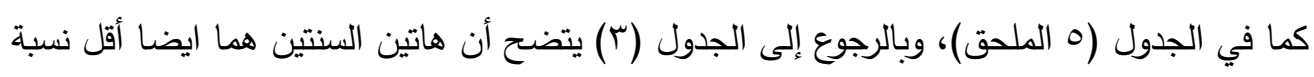

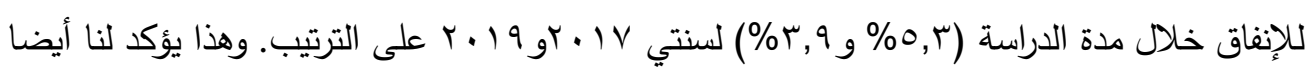

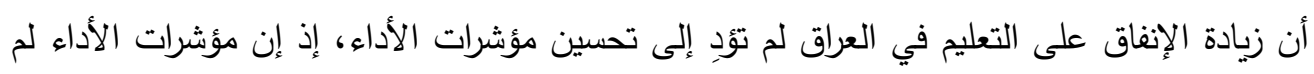

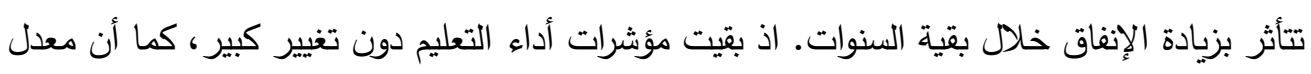

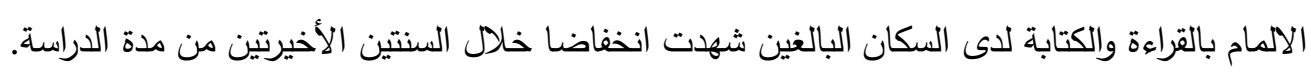

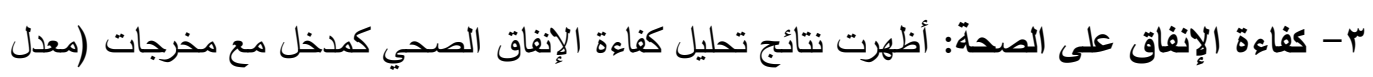

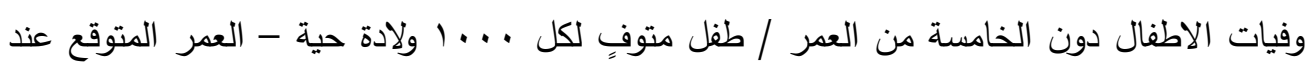

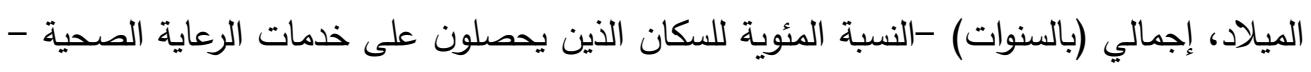

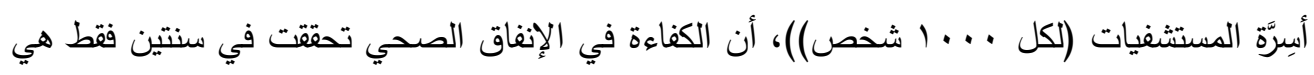

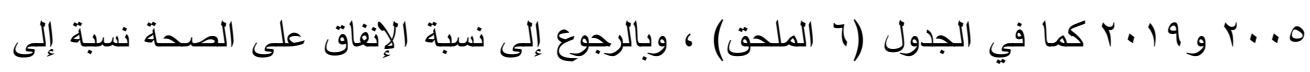


اجمالي الإنفاق يتبين أن هاتين السنتين هما من ضمن النسب المنخفضة الجدول (ع). وتؤكد لنا هذه

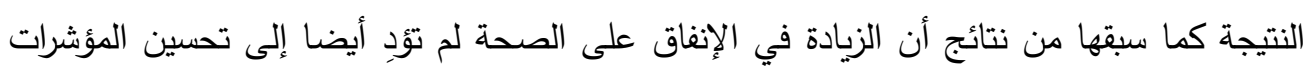

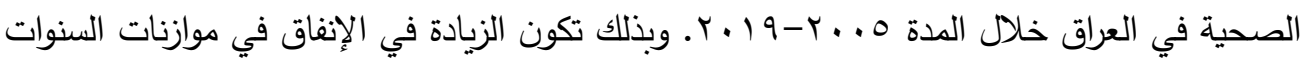
غير كفوة وهدر في الأموال، ولم تتحقق القيمة مقابل المال. مما سبق نقف على نفي فرضية البحث، أي أنه لم تتحقق الكفاءة في الإنفاق على القطاعات الثقانلاتثة في

ا- تتحقق الكفاءة التشغيلية للإنفاق بتوافر وترابط العناصر الأربعة جميعها (الاقتصاد، الكفاءة، الفعالية،

$$
\text { الانصاف). }
$$

ץ- كثفت نتائج تحليل مغلف البيانات للكفاءة التثغيلية لكل من الإنفاق الاستثماري والإنفاق على التعليم والإنفاق على الصحة أن الإنفاق على القطاعات الثلاثة لم يكن كفوءً للغالبية العظمى من موازنات الإنيات

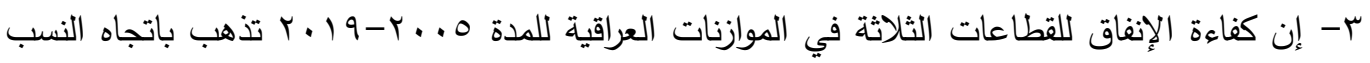
الأقل، مما يعني أن الزيادة في الإنفاق لكل من القطاعات الثثلاثة لا تؤدي إلى تحسين مؤشرات الأداء.

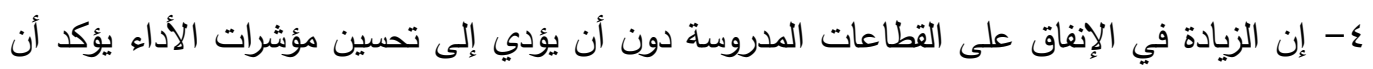

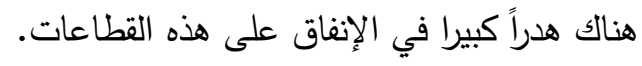

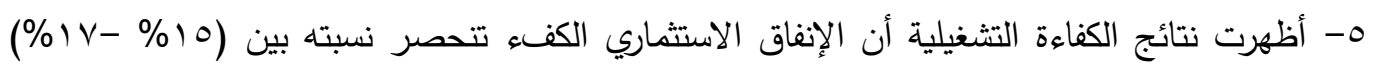

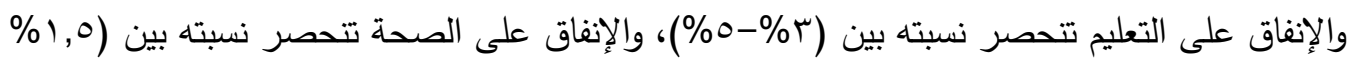

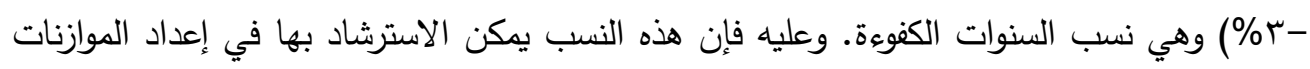
في السنوات القادمة، وشرط الزيادة مرهون بشرط رفع الأداء. المقترحات

1- تثديد إجراءات المساءلة في الأموال من اجل تحقيق الكفاءة والفاعلية وأقصى قيمة مقابل المال في الإنفاق الحكومي في العراق. r- بناءً على نتائج تحليل كفاءة بعض قطاعات الإنفاق تملك الحكومة خيار تخفيض الإنفاق بدلا من حجم

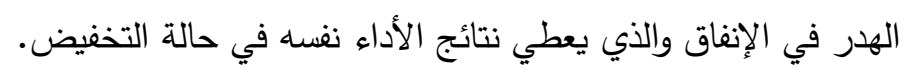

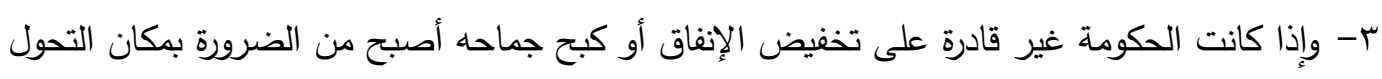
نحو موازنة البرامج والأداء. ويتمثل هذا الإصلاح الرئيس في إعداد الموازنات في الانتقال من التركيز

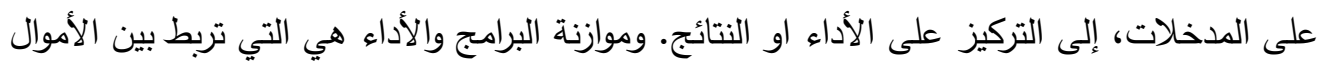

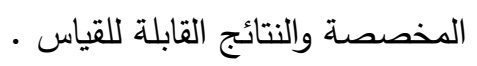




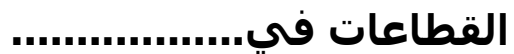 \\ علي والعباس

$$
\begin{aligned}
& \text { ع- بما أن إجراءات الموازنة في العراق تتم من (أعلى إلى أدنى) يمكن لوزارة المالية عند إعداد الموازنة أن }
\end{aligned}
$$

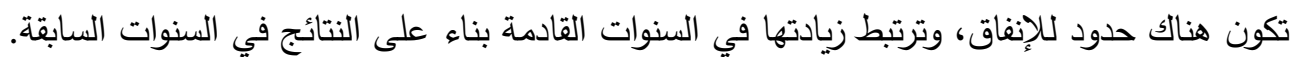

\section{Reference}

Afonso \& Kazemi, António, Mina,2016, Assessing Public Spending Efficiency in 20 OECD Countries, Working Papers, Lisbon School Of Economics \& Management .

Ali , Oday Salim,2017, Statistical Appendix of the Republic of Iraq, Arab Economic Journal, No 76-77, Central for Arab Unity Studies, Lebanon .

Ali, Oday Salim ,2019, The Impact Of Spending On The Elements Of Social Policy In Supporting Of Social Stability Study In A Sample Of The Countries Of The World For The Period 2001-2012, Journal Of Economic Sciences , Vol 14 ,No 55 , University Of Basra.

Ana , T. D. Sant \& et.al,2020, Scientific Research on the Efficiency of Public Expenditures: How and Where is it Going, International Journal of Public Administration, Volume 43, Issue 11.

Badawi, Ahmed Abu Bakr Ali ,2011,Traditional and contemporary concepts in public financial management, Economic Studies, Arab Monetary Fund, Abu Dhabi, UAE.

Bekele , Deribe ,2018, Improving Public Service Delivery And Efficiency The Case Of Addis Ababa City Fire And Emergency Prevention, A Thesis Submitted In Partial Fulfillment Of The Requirement's For The Degree Of Masters Of Art In Development Management, Addis Ababa University, Ethiopia.

Bowlin , William F., 1998, Measuring Performance: An Introduction to Data Envelopment Analysis (DEA), The Journal of Cost Analysis, Vol 15,No 2, Publisher: Taylor \& Francis.

DOI: 10.1080/08823871.1998.10462318.

Brini \& Jemmali , Riadh , Hatem ,2016, Public spending efficiency, governance, political and economic policies: Is there a substantial causal relation? Evidence from selected MENA countries, International Journal of Economics and Financial Management, Vol. 1 No. 1. www.iiardpub.org.

CDI,2015, Improving the Practice of Value for Money Assessment, Practice Paper, Centre for Development Impact,UK.

Central Statistics organization Iraq, : http://cosit.gov.iq/ar

Central Statistics organization Iraq,2011, Priority Sustainable Environment Indicators in Iraq, Environment Statistics department.

Central Statistics organization Iraq,2015, Priority Sustainable Environment Indicators in Iraq, Environment Statistics department.

Central Statistics organization Iraq,2018, Priority Sustainable Environment Indicators in Iraq, Environment Statistics department.

Scholz, Vera,2020, Value For Money, INTRAC for civil society , UK.

Cooper \& et.al , William W.,2011, Data Envelopment Analysis History, Models And Interpretations, Handbook on Data Envelopment Analysis, Springer Science.

TANMIYAT AL-RAFIDAIN (P-ISSN: 1609-591X; E-ISSN: 2664-276X) تنمية الرافدين 


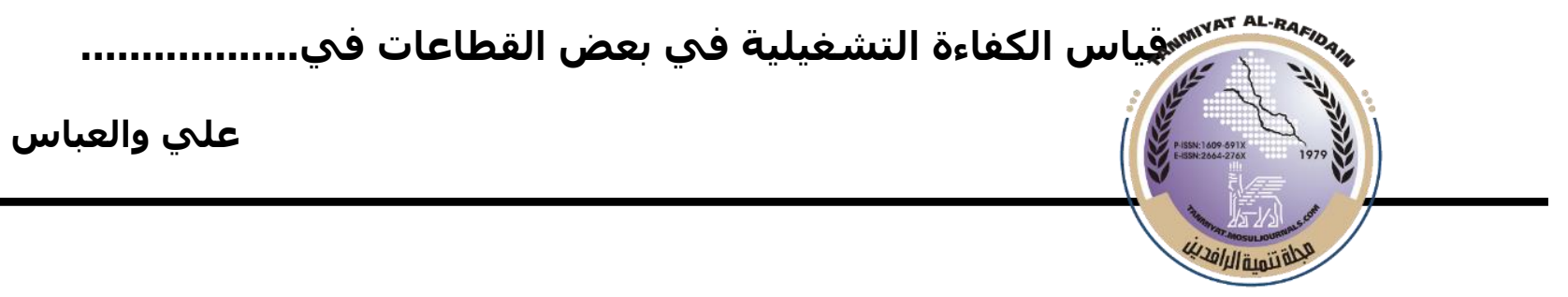

Dutu \& Sicari, Richard, Patrizio, 2020, Public Spending Efficiency in the OECD: Benchmarking Health Care, Education, and General Administration, Review of Economic Perspectives, Vol. 20, Issue 3.

Herrera \& Ouedraogo , Santiago , Abdoulaye ,2018, Efficiency of Public Spending in Education, Health, and Infrastructure An International Benchmarking Exercise, Policy Research Working Paper 8586, World Bank.

Huguenin, Jean-Marc,2012, Data Envelopment Analysis (DEA) A pedagogical guide for decision makers in the public sector, Institut de hautes études en administration publique (idheap), Switzerland.

Khalil \& Adelabu, Samihah, Salihu,2012, Model for Efficient Service Delivery in Public Service, Journal of Public Administration and Governance, Vol. 2, No. 3. https://doi.org/10.5296/jpag.v2i3.2157

Khan \& Murova , Aman, Olga,2015, Productive Efficiency of Public Expenditures: A Cross-state Study, journal State and Local Government Review , Vol. 47(3).

Mandl , Ulrike \& et.al ,2008, The Effectiveness and Efficiency of Public Spending, European Commission.

Martic \& et.al ,Milan.N,2009,Data Envelopment Analysis -Basic Models and their Utilization , journal of Organizacija, Vol .42 . No.2.

Ministry of Finance, Budget Department.

Ministry of Health, Annual Statistical Report, Various Reports.

Mujasi \& et.al , Paschal N.,2016, How efficient are referral hospitals in Uganda? A data envelopment analysis and tobit regression approach, BMC Health Services Research.

Schick ,Allen , 2007,Performance Budgeting and Accrual Budgeting: Decision Rules or Analytic Tools? , OECD Journal on Budgeting, Volume 7 - No. 2, OECD.

Statistical, Economic and Social Research and Training center for Islamic Countries (SESRIC) : https://www.sesric.org.

The Central Bank of Iraq, Annual Statistical Bulletin, Various Issues

World Bank Open Data: https://data.albankaldawli.org

Zhang \& et.al, Hongjun,2014, Dimension-Specific Efficiency Measurement Using Data Envelopment Analysis, The Journal Mathematical Problems in Engineering, Volume 2014, Hindawi Publishing Corporation. https://doi.org/10.1155/2014/247248.

TANMIYAT AL-RAFIDAIN (P-ISSN: 1609-591X; E-ISSN: 2664-276X) تنمية الرافدين 